\title{
SOME NEW DENSITY ESTIMATES FOR THE ZEROS OF THE RIEMANN ZETA-FUNCTION
}

\author{
K. RAMACHANDRA
}

1. Introduction. It is the object of this paper to prove the following

$\mathrm{T}$ h e o r e m. Let $R(\sigma, T)$ denote the rectangle $\sigma \leqq x \leqq 1,|y| \leqq T$, and $N(\sigma, T)$ the number of zeros of $\zeta(z), z=x+i y$, in it. Then we have, uniformly for $3 / 4 \leqq \sigma \leqq 1$,

$$
\begin{gathered}
N(\sigma, T) \ll\left(T^{2(1-\sigma)}+T^{1-k(4 \sigma-3)}+T^{3 k(1-\sigma) /(k(3 \sigma-1)+2(1-\sigma))}\right. \\
\left.+T^{3(1-\sigma) /(k(4 \sigma-3)+3(1-\sigma))}\right) T^{\varepsilon}
\end{gathered}
$$

where $k$ is any fixed positive integer, $\varepsilon>0$ an arbitrary constant, and the constant implied by $\ll$ depends only on $k$ and $\varepsilon$.

Taking $k=4$ we have the following

Corollary. For every $\varepsilon>0$, we have

$$
N(\sigma, T) \ll T^{2(1-\sigma)+\varepsilon},
$$

uniformly for $21 / 26 \leqq \sigma \leqq 1$.

Remark 1. We can obtain similar bounds for

$$
\sum_{\chi \bmod q} N_{\chi}(\sigma, T)
$$

where $N_{\chi}(\ldots)$ denotes the number of zeros of $L(z, \chi)$ in $R(\sigma, T)$ and also for

$$
\sum_{q \leqq Q} \sum_{\chi \bmod q}^{*} N_{\chi}(\sigma, T)
$$

(* denotes omission of improper characters) provided $q$ and $Q$ do not exceed certain function of $T$.

Remark 2. The best result so far published in the direction of the Corollary is that the inequality of the corollary is valid for $\sigma \geqq 5 / 6$ due to M. N. Huxley [1] and M. Jutila [2] independent of each other (by different methods). However, Professor M. N. Huxley has informed me (in his letter dated 15.7.1973) that he has established the truth of the above 
corollary for $\sigma \geqq 4 / 5$. I have not seen his manuscript and I hope that my method is new.

2. Notation. In the Sections $3-4$ we omit certain obvious factors like $T^{\varepsilon}$ or $T^{-\varepsilon}$ at several places and this has to be supplied by the reader at proper places.

3. An improvement of the Halász-Montgomery-Huxley estimate for the large values of a Dirichlet polynomial. So far as applications are concerned it will be sufficient to consider the case when the positive quantity $V$ (see below) satisfies $V+V^{-1}=O\left(T^{\varepsilon}\right)$ and $a_{n}=a_{n}(N)$ are complex numbers satisfying $\max \left|a_{n}\right|=O\left(N^{\varepsilon}\right)$. The definitions of $V$ and $T$ are as follows. We are given a finite set of distinct complex numbers $s_{r}=\sigma_{r}+i t_{r}(r=1,2, \ldots, R)$. We put $\min \sigma_{r}=\sigma, \max t_{r}-\min t_{r}+$ $20=T$ and impose the condition $\min _{r \neq r^{\prime}}\left|t_{r}-t_{r^{\prime}}\right| \geqq \log ^{2} T$. We are given a Dirichlet polynomial

$$
f(z)=\sum_{N \leqq n \leqq 2 N} a_{n} n^{-z} .
$$

We suppose that for $r=1,2, \ldots, R$ we have $\left|f\left(s_{r}\right)\right| \geqq V$ and seek to find an upper bound for $R$. We shall also assume that $\sigma \geqq 3 / 4$ and $\sigma_{r} \leqq 1$ for all $r$.

For suitable complex numbers $\eta_{r}$ of absolute value 1, we have

$$
\begin{aligned}
R V & \leqq \sum_{r} \eta_{r} \sum_{n} a_{n} n^{-s_{r}}=\sum_{n} a_{n} n^{-\sigma} \sum_{r} \eta_{r} n^{\sigma-s_{r}} \\
& \ll G^{1 / 2}\left(\sum_{n} b_{n} \sum_{r, r^{\prime}} \eta_{r} \bar{\eta}_{r^{\prime}} n^{\left.2 \sigma-s_{r}-\overline{s_{r^{\prime}}}\right)^{1 / 2}},\right.
\end{aligned}
$$

where

$$
G=\sum_{n}\left|a_{n}\right|^{2} n^{-2 \sigma}
$$

and

$$
b_{n}=e^{-n / 2 N}-e^{-n / N}=e^{-n / N}\left(e^{n / 2 N}-1\right) \geqq e^{-2 N / N}(n / 2 N) \geqq \frac{1}{2} e^{-2}
$$

in the relevant range for $n$. We can now take the sum over all $n$ from 1 to infinity in the bracket. We observe that $\sum b_{n} \ll N$ and so separating the terms with $r=r^{\prime}$ and $r \neq r^{\prime}$ we get the estimate

$$
\begin{aligned}
R V & \ll G^{1 / 2}\left[R N+\sum_{r \neq r^{\prime}}(2 \pi i)^{-1} \int_{2-i \infty}^{2+i \infty} \zeta\left(s_{r}+\bar{s}_{r^{\prime}}\right.\right. \\
& \left.-2 \sigma+w) \Gamma(w)\left((2 N)^{w}-N^{w}\right) d w\right]^{1 / 2} .
\end{aligned}
$$


We can regard the inner sum as $\sum_{r^{\prime}} \sum_{r \neq r^{\prime}}$ and pick out $r^{\prime}=r_{0}$ say, for which the inner sum over $r$ is maximum. Thus we get the estimate

$$
R V \ll N^{1 / 2-\sigma} R^{1 / 2}\left[N^{1 / 2}+\left(\sum_{r \neq v_{0}}\left|\int_{2-i \infty}^{2+i \infty}(\ldots) d w\right|\right)^{1 / 2}\right] .
$$

Next we impose the condition that $N$ shall not exceed a fixed power of $T$ and move the line of integration to $u$ given by $(w=u+i v) \sigma_{r}+\sigma_{r_{0}}$ $2 \sigma+u=-(\log T)^{-1}$, i.e. $u=2 \sigma-\sigma_{r}-\sigma_{r_{0}}-(\log T)^{-1}$. It is easy to check that $-\frac{1}{2}-(\log T)^{-1} \leqq u \leqq-(\log T)^{-1}$. We next apply the functional equation for $\zeta(z)$ in the form $\zeta(z)=\psi(z) \zeta(1-z)$ to get

$$
\psi\left(s_{r}+\bar{s}_{r_{0}}-2 \sigma+w\right) \zeta\left(1-s_{r}-\bar{s}_{r_{0}}+2 \sigma-w\right)
$$

in place of $\zeta(\ldots)$ in the integrand. We next write the series for $\zeta(\ldots)$, with $Y=T^{1 / k}$ ( $k$ a fixed positive integer), in the form

$$
\zeta\left(1-s_{r}-\bar{s}_{r_{0}}+2 \sigma-w\right)=\sum_{n \geqq Y}+\sum_{n<Y}
$$

and denote the corresponding integrals by $I_{1}=I_{1}\left(r, r_{0}\right)$ and $I_{2}=$ $=I_{2}\left(r, r_{0}\right)$. In $I_{2}$ we next move the line of integration to $u$ given by $\quad 1-\sigma_{r}-\sigma_{r_{0}}+2 \sigma-u=1 / 2$, i.e. $u=1 / 2+2 \sigma-\sigma_{r}-\sigma_{r_{0}}$. Observe that $0 \leqq u \leqq 1 / 2$. We now break off the integrals $I_{1}$ and $I_{2}$ at $v= \pm \log ^{3} T$ with a small error. This will enable us to prove Lemmas 1 and 2 below. (Of course we have to use Hölder's inequality and a theorem of Davenport; see Theorem 1 of [3]).

$\mathrm{L}$ e $\mathrm{m} \mathrm{m}$ a 1 . We have

$$
\sum_{r \neq r_{0}}\left|I_{1}\left(r, r_{0}\right)\right| \ll R^{1-1 / 2 k} T^{1 / 2}
$$

$\mathrm{L}$ e $\mathrm{m} \mathrm{m}$ a 2. We have

$$
\sum_{r \neq r_{0}}\left|I_{2}\left(r, r_{0}\right)\right| \ll N^{1 / 2} T^{1 / 2 k} R^{1-1 / 2 k} .
$$

Using these two lemmas we get

$\mathrm{L}$ e $\mathrm{mm}$ a 3 . We have

$$
R \ll N^{1-\sigma} R^{1 / 2}+R^{1-1 / 4 k} N^{1 / 2-\sigma} T^{1 / 4}+N^{3 / 4-\sigma} T^{1 / 4 k} R^{1-1 / 4 k} .
$$

This gives as a corollary

$\mathrm{L}$ e $\mathrm{mma}$. If $T \geqq 20$, then

$$
R \ll N^{2(1-\sigma)}+T^{k} N^{-2 k(2 \sigma-1)}+T N^{-k(4 \sigma-3)} .
$$

We now assume $T \geqq 200$ and break up the set of points into at most $1+T T_{0}^{-1}$ sets where in each set we can take $T_{0}$ in place of $T$ (assuming that $T_{0} \geqq 20$ ). We then have 
L e m m a 5 . If $T \geqq 200$ and $T_{0} \geqq 20$, then

$$
R \ll\left(N^{2(1-\sigma)}+T_{0}^{k} N^{-2 k(2 \sigma-1)}+T_{0} N^{-k(4 \sigma-3)}\right)\left(1+T T_{0}^{-1}\right) .
$$

Let $T_{1}$ and $T_{2}$ be defined by

$$
\begin{aligned}
& T_{1}=\left(N^{2(1-\sigma)+2 k(2 \sigma-1)}\right)^{1 / k}, \\
& T_{2}=N^{2(1-\sigma)+k(4 \sigma-3)} .
\end{aligned}
$$

Then since $N^{2(1-\sigma)}$ in the lemma dominates if $T_{0} \leqq \min \left(T_{1}, T_{2}\right)$ we have by taking $T_{0}=\min \left(T_{1}, T_{2}\right)$

L e $\mathrm{m} \mathrm{m}$ a 6 . We have

$$
R \ll N^{2(1-\sigma)}\left(1+T_{1}^{-1} T+T_{2}^{-1} T\right) .
$$

Proof. The condition $\min \left(T_{1}, T_{2}\right) \geqq 20$ can be dropped since otherwise the lemma states the trivial bound $R \ll T$.

Lemma 6 gives

L e $\mathrm{m} \mathrm{ma} 7$. We have for $T \geqq 200$ and for $N$ not exceeding a fixed power of $T$

$$
R \ll N^{2(1-\sigma)}+T N^{4-6 \sigma-2(1-\sigma) / k}+T N^{-k(4 \sigma-3)} .
$$

Remark. The limiting case $k \rightarrow \infty$ here is due to Huxley [1], who deduced it from a result of Montgomery. Our method is somewhat more complicated.

4. The density estimates. Further work to prove the theorem is a device due to Montgomery with sharpenings due to Jutila explained in [1]. We repeat it to some extent. Let $\delta>0$ be a small constant and let $F(z)=\zeta(z) M(z)-1$, where

$$
M(z)=\sum_{n \leqq T^{\delta}} \mu(n) n^{-z}
$$

It is clear that if $\varrho=\beta+i \gamma$ is a zero of $\zeta(z)$ in $R(\sigma, T)$ then $|F(\varrho)|=1$. Let the sequence $\left\{a_{n}\right\}$ be defined by

$$
F(z)=\sum a_{n} n^{-z},
$$

where $\operatorname{Re} z=x>1$. Clearly $a_{n}=0$ if $n \leqq T^{\delta}$. Also the number of zeros with $|\gamma| \leqq \log ^{2} T$ is $O\left(\log ^{3} T\right)$. We first select, (observe that $N(0, T+1)-N(0, T)=O(\log T)), \quad$ a maximal subset of zeros in $R(\sigma, T)$ with the properties

$$
\begin{gathered}
|\gamma| \geqq \log ^{2} T, \\
\min _{\gamma \neq \gamma^{\prime}}\left|\gamma-\gamma^{\prime}\right| \geqq \log ^{2} T .
\end{gathered}
$$


It suffices to estimate the number of these zeros. For these zeros consider the identity

$$
\sum_{n \geqq T^{\delta}} a_{n} n^{-\varrho} e^{-n / Y}=(2 \pi i)^{-1} \int_{2-i \infty}^{2+i \infty} F(\varrho+w) \Gamma(w) Y^{w} d w .
$$

By moving the line of integration to $u$ given by $u+\beta=1 / 2$ and then using

$$
\int_{|t| \leqq T}|\zeta(1 / 2+i t)|^{4} d t \ll T \log ^{4} T
$$

(or its consequence

$$
\sum_{\left|t_{r}\right| \leqq T}\left|\zeta\left(1 / 2+i t_{r}\right)\right|^{4} \ll T \log ^{10} T
$$

in an obvious notation) and choosing $Y=T^{1 / 2+10 \delta}$, we see that

$$
\left|\sum_{T^{1 / 2+20 \delta} \geqq n \geqq T^{\delta}} d_{n} n^{-\varrho}\right| \geqq(\log T)^{-8}
$$

(with $d_{n}=a_{n} e^{-n / Y}$ and $\left|d_{n}\right| \leqq d(n)$ ) for all such zeros with the exception of at most $T^{2(1-\sigma)+\eta}$ zeros where $\eta=\eta(\delta)$ tends to zero as $\delta$ tends to zero. From this we get a zero detecting device of the form

$$
\left|\sum_{U \leqq n \leqq 2 U} d_{n} n^{-0}\right| \geqq(\log T)^{-10} \quad\left(T^{\delta} \leqq U \leqq T^{1 / 2+20 \delta}\right)
$$

where $U$ is the same for all zeros under consideration except for a proportion $1-(\log T)^{-12}$. If $U>T^{1 / 2}$ then we use the square of $\sum d_{n} n^{-z}$ as the zero detecting function and use the results of Section 3. Otherwise we have $T^{1 /(m+1)} \leqq U \leqq T^{1 / m}$ with $2 \leqq m \leqq 2 \delta^{-1}$. We use

$$
\left(\sum_{U \leqq 2 U} d_{n} n^{-z}\right)^{l}
$$

as the zero detecting function with $l=m+1$ or $l=m$ according as $T^{1 /(m+1)} \leqq U \leqq T^{\lambda}$ or $T^{\lambda}<U \leqq T^{1 / m}$, where $\lambda$ is a positive parameter to be chosen to the maximum advantage. (Of course the zero detecting function is of the form $\sum_{N \leqq n \leqq C N}$ where $C$ is a large constant but we can pass to a function of the form $\sum_{N \leqq n \leqq 2 N}$ or apply a trivial modification of the results of Section 3). We get

$$
\begin{gathered}
N(\sigma, T) \ll T^{2(1-\sigma)}+T^{1-k(4 \sigma-3)}+T^{5-6 \sigma-2(1-\sigma) / k} \\
+\min \left(T^{2 \lambda(m+1)(1-\sigma)}+T^{1-k \lambda m(4 \sigma-3)}+T^{1-\lambda m(6 \sigma-4+2(1-\sigma) / k)}\right. \\
\ll T^{2 \lambda_{1}(m+1)(1-\sigma)}+T^{2 \lambda_{2}(m+1)(1-\sigma)}+T^{2(1-\sigma)}+T^{1-k(4 \sigma-3)}+T^{5-6 \sigma-2(1-\sigma) / k},
\end{gathered}
$$

where 


$$
\begin{gathered}
\lambda_{1}\{2(m+1)(1-\sigma)+k m(4 \sigma-3)\}=1 \\
\lambda_{2}\{2(m+1)(1-\sigma)+m(6 \sigma-4+2(1-\sigma) / k)\}=1 .
\end{gathered}
$$

Now $(m+1) \lambda_{1}$ and $(m+1) \lambda_{2}$ are decreasing as $m$ increases so that we can take $m=2$ to get the bound in all cases. This leads to the theorem stated in the introduction. (In the computation of the minimum over $\lambda$ it is convenient to use a lemma of van der Corput as modified by B. R. Srinivasan (see Lemma 4 of [5])).

\section{Added in proof}

Recently Professor M. Jutila has proved the Density hypothesis for $\sigma \geq$ 11/14 and the $q$ and $Q$ Density hypothesis for $\sigma \geq 4 / 5$; see his papers "zero-density estimates for $L$-functions I and II" (to appear). He has also simplified the proofs of Huxley's Density results.

\section{References}

[1] Huxley, M. N.: On the difference between consequtive primes. - Invent. Math. 15, 1972, 164-170.

[2] JutilA, M.: On a density theorem of H. L. Montgomery for $L$-functions. Ann. Acad. Sci. Fenn. Ser. A I 520, 1972, 1-13.

[3] Montgomery, H. L.: Mean and large values of Dirichlet polynomials. Invent. Math. 8, 1969, 334-345.

[4] -》- Zeros of $L$-functions. - Invent. Math. 8, 1969, 346-354.

[5] SRInivasan, B. R.: On van der Corput's and Nieland's results on the Dirichlet divisor problem and the circle problem. - Proc. Nat. Acad. Sci. India, Sect. A 28, 1962, 732-742.

Tata Institute of Fundamental Research

School of Mathematics

Colaba, Bombay-5

India

Received 3 February 1975 